\title{
Model predictive allocation control for leg-wheel mobile robot on loose soil considering wheel dynamics
}

\author{
Takatsugu Oda $^{1}$ Hiroki Yoshikawa $^{1}$ Naoki Shibata $^{1} \quad$ Kenichiro Nonaka $^{1} \quad$ Kazuma Sekiguchi $^{1}$ \\ ${ }^{1}$ Mechanical systems engineering, Tokyo city university, Japan, \{knonaka, ksekiguc $\}$ @tcu.ac.jp
}

\begin{abstract}
For the planetary exploration rover, to cope with the layer of heterogeneous superficial deposits called regolith is important so as to achieve designed traction. In order to consider effects of wheel motion, model predictive allocation control is proposed. To cope with complex terramechanics in MPC (model predictive control), the identification technique is introduced; the proposed MPC is formed as a linear optimization problem. The rover model and terramechanics are described using modelica and simulate to evaluate the performance of the proposed method. The suppressed of superfluous slip and enhancement of traction performance is numerically shown.
\end{abstract}

Keywords: Terramechanics, Allocation control, Model predictive control, Model identification.

\section{Introduction}

Developments of autonomous robots are widely studied to apply for various environments in which human cannot work. Especially for planetary exploration, exploration rovers are generally adopted, because these robots can move planetary surface efficiently (Weisbin et al., 1997). However, on many planets, planetary surface is covered with a layer of heterogeneous superficial deposits called regolith; wheels might slip and not generate desired forces on these soil. In addition, since the deformable loose soil on which wheels passed are compressed, the terrain property (e.g. soil density, resistance force etc.) would change (Senatore and Sandu, 2011). Then, for autonomous robots equipped with drive wheel, wheel control technique is important to suppress the slip and wheel sinkage.

Based on the terramechanics which deal with the interaction of the wheel and soil (Taheri et al., 2015), many vehicle control method is studied. In order to enhance traction and energy performance, dual-criteria objective function for control allocation is proposed in (Iagnemma and Dubowsky, 2004). For the traction control of the rover, PI controller with slip ratio estimator (Yoshida and Hamano, 2002) and robust adaptive fuzzy control strat- egy (Zhengcai and Yang, 2014) are developed. In addition, MPC (model predictive control) which could consider the dynamics and constraint explicitly is applied to planetary rovers (Krenn et al., 2013). However, this method adopts the linearization technique to consider complex model and does not consider muli-pass effect.

In this paper, the model predictive allocation control for multi-wheel rovers considering the terramechanics including the multi-pass effect is proposed. The wheel dynamics is explicitly considered using MPC, dynamically infeasible allocated solution which static optimization method might have is avoided. To consider complex terramechanics model in linear MPC, black box identification technique is introduced; for identification, the rover model is operated in only linear region of terramechanics. Effectiveness of the model predictive allocation control is verified through numerical simulation using modelica; suppression of superfluous slip and enhancement of traction performance is shown. Note that to focus on the longitudinal motion, we assume lateral motion of the rover could be sufficiently small.

\section{Vehicle and wheel model}

\subsection{Leg-wheel mobile robot}

In this paper, a leg-wheel mobile robot consists of a body and limbs, wheels, as depicted in Fig. 1 is considered. By using redundancy of limbs equipped with six joint, the desired wheel arrangement is achieved (Yoshikawa et al., 2017). The wheels could drive independently and generate tire force based on terramechanics which express wheel-soil interaction mechanisms.

\subsection{Wheel model based on terramechanics}

We introduce the wheel model based on terramechanics (Senatore and Sandu, 2011) into the robot model in order to simulate the force and moment characteristics of the tire on a deformable terrain (Yoshikawa et al., 2017). Roughly, in this model, generated force of each wheels are calculated by integrating normal $\sigma$ and shear $\tau_{x}$ stress distribution model which described in Fig. 2. 


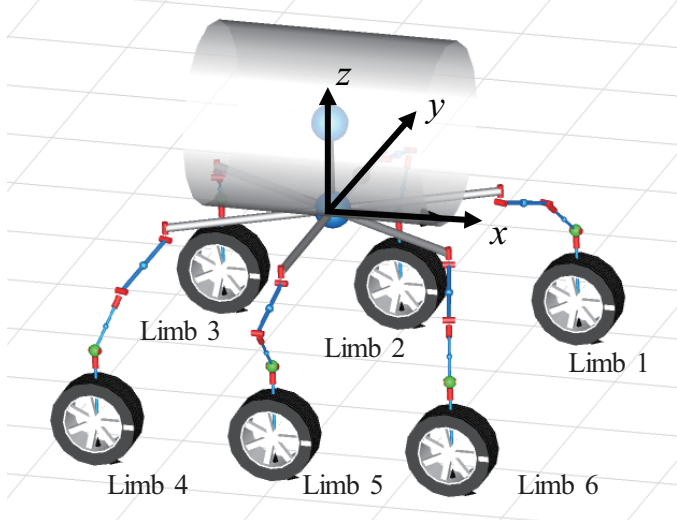

Figure 1. Leg-wheel mobile robot with six joints of each limb.

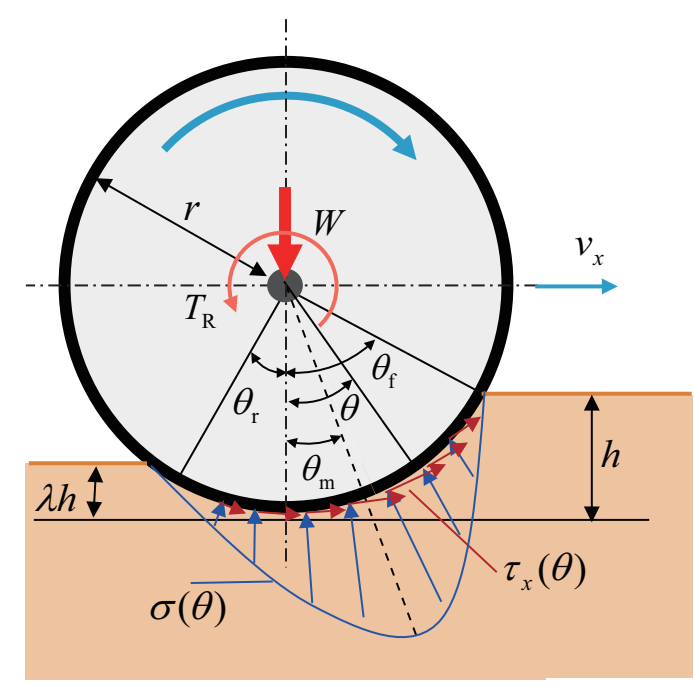

Figure 2. Normal and shear stress distribution concept of terramechanics while rolling.

The maximum stress angle is empirically estimated as following a linear function of the slip ratio $\kappa$ and the entry angle $\theta_{\mathrm{f}}$ :

$$
\theta_{\mathrm{m}}=\left(a_{0}+a_{1} \kappa\right) \theta_{\mathrm{f}},
$$

where $a_{0}$ and $a_{1}$ are constant parameter. The slip ratio is explained as follows with the wheel radious $r$, angular velocity $\omega$ and translational velocity $v_{x}$ :

$$
\kappa=\frac{r \omega-v_{x}}{\max \left(r \omega, v_{x}\right)},
$$

The empirical normal stress distribution based on Reece's formula are expressed as follows:

$$
\begin{aligned}
\sigma(\theta) & = \begin{cases}\sigma_{\mathrm{f}}(\theta) & \left(\theta_{\mathrm{m}} \leq \theta<\theta_{\mathrm{f}}\right), \\
\sigma_{\mathrm{r}}(\theta) & \left(\theta_{\mathrm{r}}<\theta \leq \theta_{\mathrm{m}}\right),\end{cases} \\
\sigma_{\mathrm{f}}(\theta) & =\left(c k_{c}^{\prime}+\rho b k_{\phi}^{\prime}\right)\left[\frac{r}{b}\left(\cos \theta-\cos \theta_{\mathrm{f}}\right)\right]^{n}, \\
\sigma_{\mathrm{r}}(\theta) & =\left(c k_{c}^{\prime}+\rho b k_{\phi}^{\prime}\right) \\
& {\left[\frac{r}{b}\left(\cos \left\{\theta_{\mathrm{f}}-\frac{\theta-\theta_{\mathrm{r}}}{\theta_{\mathrm{m}}-\theta_{\mathrm{r}}}\left(\theta_{\mathrm{f}}-\theta_{\mathrm{m}}\right)\right\}-\cos \theta_{\mathrm{f}}\right)\right]^{n}, }
\end{aligned}
$$

where the Bekker-Reece sinkage exponent $n$ is a linear function of slip ratio; $n=n_{0}+n_{1}|\kappa| . \quad b$ is wheel width and $c$ is cohesion stress of the soil, $\rho$ is soil density, $k_{c}^{\prime}$ is cohesion related soils parameter, $k_{\phi}^{\prime}$ is angle internal friction related soil parameter, respectively.

The empirical shear stress distribution introduced by Janosi and Hanamoto is widely used (Taheri et al., 2015):

$$
\begin{aligned}
\tau_{x} & =(c+\sigma(\theta) \tan \phi)\left(1-e^{-j_{x}(\theta) / k_{x}}\right), \\
j_{x}(\theta) & =r\left[\theta_{\mathrm{f}}-\theta-(1-\kappa)\left(\sin \theta_{\mathrm{f}}-\sin \theta\right)\right],
\end{aligned}
$$

where $j_{x}$ is the shear displacement in longitudinal direction and $\phi$ is angle of internal friction of the soil, $k_{x}$ is shear deformation modulus in the longitudinal direction, respectively.

In order to express the multi-pass effect, as which the effect of repetitive loading of deformable soils is expressed, some parameters are modified; the modified soil density $\rho_{\mathrm{p}}$, soil cohesion $c_{\mathrm{p}}$ and shear deformation modulus in the longitudinal direction $k_{x p}$ are formulated as follows:

$$
\begin{aligned}
\rho_{\mathrm{p}} & =\rho\left(1+\left(1-e^{\frac{-k_{0}}{k_{1}}}\right) k_{2}+k_{3} n_{\mathrm{p}}\right), \\
c_{\mathrm{p}} & =c\left(1+\left(1-e^{\frac{-k_{0}}{k_{1}}}\right) k_{2}+k_{3} n_{\mathrm{p}}\right), \\
k_{x \mathrm{p}} & =k_{x}\left(1-\left(1-e^{\frac{-k_{0}}{k_{1}}}\right) k_{2}-k_{3} n_{\mathrm{p}}\right),
\end{aligned}
$$

where $\kappa_{0}$ is slip ratio of previous pass and $n_{\mathrm{p}}$ is number of passes, $k_{1}, k_{2}, k_{3}$ are constant parameters, respectively.

The vertical force $F_{z}$ and the longitudinal force $F_{x}$, rolling resistance torque $T_{\mathrm{R}}$ are calculated by integrating normal $\sigma$ and shear $\tau_{x}$ stress distribution as follows:

$$
\begin{aligned}
& F_{z}=r b \int_{\theta_{\mathrm{r}}}^{\theta_{\mathrm{f}}}\left\{\tau_{x}(\theta) \sin \theta+\sigma(\theta) \cos \theta\right\} \mathrm{d} \theta, \\
& F_{x}=r b \int_{\theta_{\mathrm{r}}}^{\theta_{\mathrm{f}}}\left\{\tau_{x}(\theta) \cos \theta-\sigma(\theta) \sin \theta\right\} \mathrm{d} \theta, \\
& T_{\mathrm{R}}=r^{2} b \int_{\theta_{\mathrm{r}}}^{\theta_{\mathrm{f}}} \tau_{x}(\theta) \mathrm{d} \theta .
\end{aligned}
$$

Note that firstly in order to accord calculated load $F z$ to given load $W$, i.e. $W=F z$, Eq. (11) is solved with respect to sinkage $h$, and then Eqs. (12)(13) are calculated with the calculated sinkage.

\section{Wheel model identification}

Because the original wheel model based on terramechanics Eqs. (11)-(13) are too complex to consider in controller design, we extract a more simple model. In this paper, we adopt black-box identification technique; tire dynamics is expressed as a first order delay system and tire model is expressed as a static linear function, as shown in Fig. 3. Because of dependent properties, we make and refer the LUT (look up table) for each parameter. 
Wheel dynamics is expressed as follows:

$$
G_{\mathrm{w}}(s)=\frac{K}{T_{1} s+1}
$$

where the gain $K\left(T_{\mathrm{w}}, W, \kappa_{0}, n_{\mathrm{p}}\right)$ and the time constant $T_{1}\left(T_{\mathrm{w}}, W\right)$ are expressed using the LUT. On the deformable terrain, small slip ratio region called linear region is mainly used; a linear tire model is adopted. The tire model, which express relationship between slip ratio $\kappa$ and longitudinal force $F_{x}$, is expressed as follows:

$$
F_{x}=a \kappa+b
$$

where $a\left(W, \kappa_{0}, n_{\mathrm{p}}\right)$ and $b(W)$ are parameter using the LUT.

\section{Control methods}

\subsection{Conposition and guidance control}

In this paper, the proposed method consists of guidance controller and model predictive allocation control. In the guidance controller, the vehicle total traction force is calculated based on the vehicle position and longitudinal velocity. Then, in the allocation controller, the vehicle total traction force is allocated among the wheels.

In the guidance controller, to achieve the target vehicle position $x_{r}$ and velocity $v_{x, r}$, a linear quadratic regulator is adopted. The vehicle total traction force $F_{x \text {,all }}$ is desinged as follows:

$$
F_{x, \text { all }}=-K_{\mathrm{LQ}}\left[\begin{array}{l}
x-x_{r} \\
v_{x}-v_{x, r}
\end{array}\right]
$$

where $K_{\mathrm{LQ}}$ is the optimal feedback coefficient matrix and given by solving the continuous time algebraic Riccati equation.

\subsection{Model predictive allocation control}

Control allocation could be solved without considering any dynamics, because it is essentially static optimal problem. However, without considering the dynamics of the wheel, optimal solution might not be achievable. To deal with this problem, a model predictive allocation control is proposed.

In this control allocation, state $\xi=\left[\kappa_{1}, \cdots, \kappa_{6}\right]^{\mathrm{T}}$ and input $u=\left[T_{\mathrm{w}, 1}, \cdots, T_{\mathrm{w}, 6}\right]^{\mathrm{T}}$ are introduced to formulate the proposed method as follows:

$$
\min _{u(i \mid k)} \sum_{i=1}^{N+1}\left(\left\|\xi(i \mid k)-\xi_{r}(i)\right\|_{Q}^{2}+\|u(i \mid k)\|_{R}^{2}\right)
$$

subject to

$$
\begin{aligned}
\xi(i+1 \mid k) & =A \xi(i \mid k)+B u(i \mid k), \\
F_{x, \text { all }}(i \mid k) & =\sum_{i=1}^{6} F_{x, i}\left(\kappa_{i}(i \mid k), f_{z, i}, \kappa_{0, i}, n_{p, i}\right), \\
|\xi(i \mid k)| & \leq \bar{\xi} \\
|u(i \mid k)| & \leq \bar{u}
\end{aligned}
$$

Eq. (17a) express the index function and $Q, R>0$ are weight matrix and $N$ is predicive horizon. $\xi_{r}$ is reference state which calculated by assuming equal traction force distribution. Eq. (17b) is the discretized statespace representation model of Eq. (14). Eq. (17c) is the condition for achieve vehicle total traction force $F_{x \text {,all }}$. Eqs. $(17 \mathrm{~d})(17 \mathrm{e})$ are the region of state and input expressed by bound of state $\bar{\xi}$ and input $\bar{u}$. Model predictive allocation control Eq. (17) is solved every sampling times with the condition $\xi(1 \mid k)=\xi(k)$; the first element of imput $u(1 \mid k)$ is adopted as applied input at $t$.

\section{Numerical simulation}

\subsection{Conditions}

In order to verify the effectiveness of the proposed method, we model the leg-wheel mobile robot with wheel model based on terramechanics using modelica (see more details in (Yoshikawa et al., 2017)), and apply the model predictive allocation control.

Some environmental parameters including soil parameters are set, so as to simulate robot behavior in lunar environment (Ishigami et al., 2007; Senatore and Sandu, 2011). The vehicles are commanded to run at constant speed $0.4 \mathrm{~m} / \mathrm{s}$. A static control allocation control technique, which does not consider any dynamics, with PI wheel velocity controller is introduced as a comparing method; same guidance controller Eq. (16) is adopted in the both (Prop. and Comp.) controller. Then, the effectiveness of considering wheel dynamics in control allocation is clearly evaluated.

\subsection{Results and discussions}

Simulation results are shown in Fig. 4; we especially show the response of limb 1-3, because of symmetric leg position. As Fig. 4(a) indicate, both methods almost achieve the target velocity. The allocated force in compared method is not achieved because of discontinuous change; on the other hands, using the proposed method, which considers the wheel dynamics, the wheel torques change continuously. Then, wheel torques of the proposed method are smaller than that of comparing method and is generated efficiently, as depicted in Fig. 4(b). Result of the acceleration, Fig. 4(c) shows increased vertical load of rear limb. This load shift leads to the bias of the slip ratio, as depicted in Fig. 4(d). Neither considering 


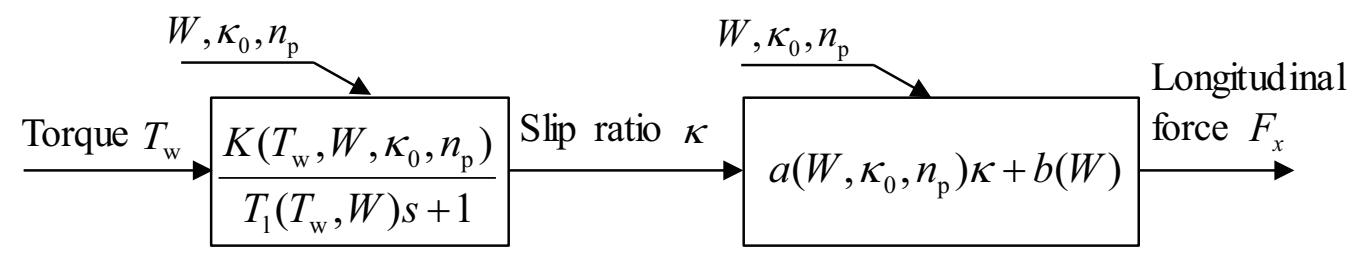

Figure 3. Structure of identified wheel model.

dynamics or not, the low load wheels are commanded large slip ratio so as to equalize tire forces. The longitudinal force distribution is, however, different from load distribution, because of considering the muti-pass effect and the compressed soil property, as depicted in Fig. 4(e). This slip ratio distributions should be suppressed, because slip ratio occurs the sinkage as Fig. 4(d)(f) shows. Then, the amounts of sinkage are reduced in the proposed controller by reducing the slip ratio distribution shown in Fig. $4(\mathrm{~g})$. Moreover, from the point of view of the effect of considering wheel dynamics, desired acceleration in LQR controller is achieved, because the proposed controller allocates facile longitudinal force; then, overshooting phenomenon of vehicle velocity is removed. It is why total slip ratio value is reduced.

\section{Conclusion}

In this paper, to consider wheel dynamics in allocation control, model predictive control method is adopted. To consider the complex and nonlinear terramechanics including multi-pass effect in linear MPC, identified wheel model consists of wheel dynamics and tire model is introduced. Numerical simulation is conducted using modelica, the effectiveness of the proposed control method is clearly shown.

Future direction of this study is to consider the lateral motion and to evaluate the traction efficiency.

\section{References}

Karl Iagnemma and Steven Dubowsky. Traction control of wheeled robotic vehicles in rough terrain with application to planetary rovers. The international Journal of robotics research, 23(10-11):1029-1040, 2004.

Genya Ishigami, Akiko Miwa, Keiji Nagatani, and Kazuya Yoshida. Terramechanics-based model for steering maneuver of planetary exploration rovers on loose soil. Journal of Field robotics, 24(3):233-250, 2007.

Rainer Krenn, Andreas Gibbesch, Giovanni Binet, and Alberto Bemporad. Model predictive traction and steering control of planetary rovers. 2013.

C Senatore and C Sandu. Off-road tire modeling and the multipass effect for vehicle dynamics simulation. Journal of Terramechanics, 48(4):265-276, 2011.
Sh Taheri, C Sandu, S Taheri, E Pinto, and D Gorsich. A technical survey on terramechanics models for tire-terrain interaction used in modeling and simulation of wheeled vehicles. Journal of Terramechanics, 57:1-22, 2015.

CR Weisbin, D Lavery, and G Rodriguez. Robotics technology for planetary missions into the 21st century. 1997.

Kazuya Yoshida and Hiroshi Hamano. Motion dynamics and control of a planetary rover with slip-based traction model. In Unmanned Ground Vehicle Technology IV, volume 4715, pages 275-287. International Society for Optics and Photonics, 2002.

Hiroki Yoshikawa, Takatsugu Oda, Kenichiro Nonaka, and Kazuma Sekiguchi. Modeling and simulation of wheel driving systems based on terramechanics for planetary explanation rover using modelica. In Proceedings of the 12th International Modelica Conference, number 132, pages 901907. Linköping University Electronic Press, 2017.

Li Zhengcai and Wang Yang. Robust adaptive fuzzy control for planetary rovers while climbing up deformable slopes with longitudinal slip. Advances in Aerospace Engineering, 2014, 2014. 


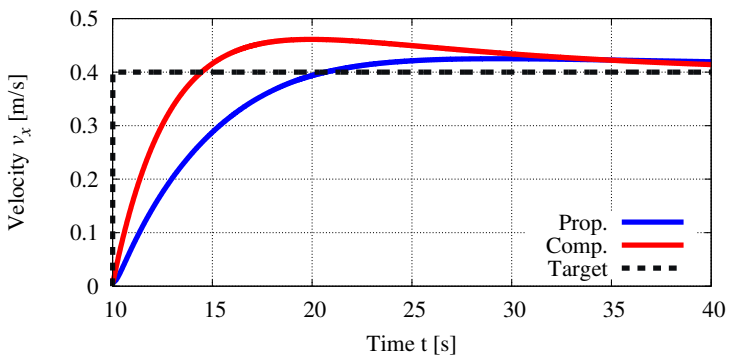

(a) Vehicle longitudinal velocity $v_{x}$.

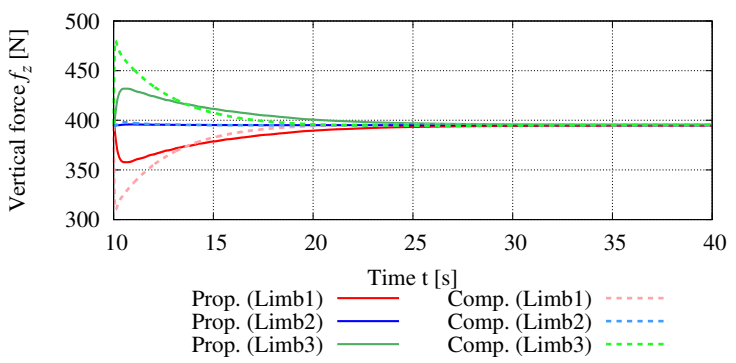

(c) Vertical force $F_{z}$.

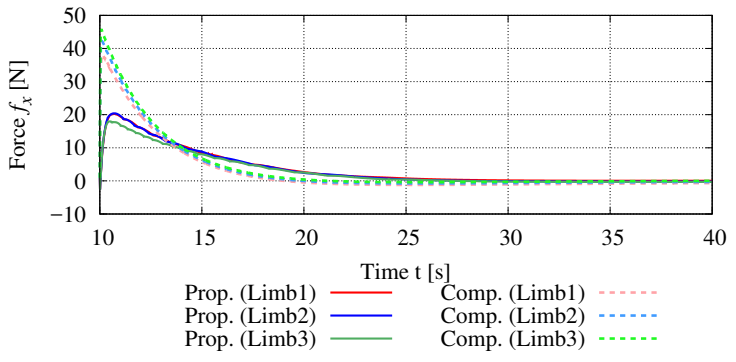

(e) Longitudinal force $F_{x}$.

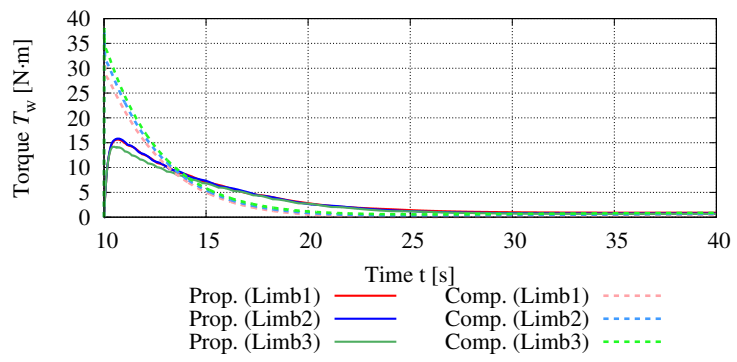

(b) Wheel torque $T_{\mathrm{w}}$.

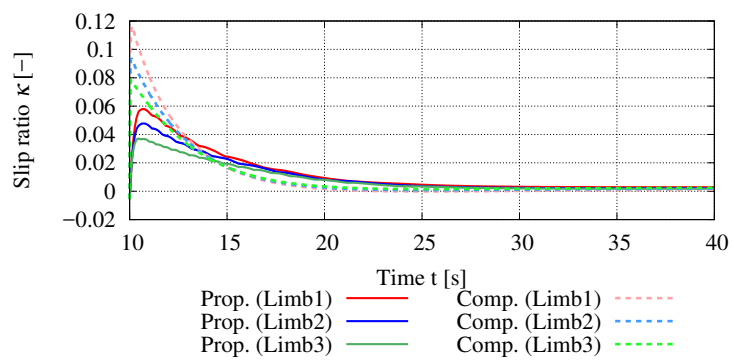

(d) Slip ratio $\kappa$.

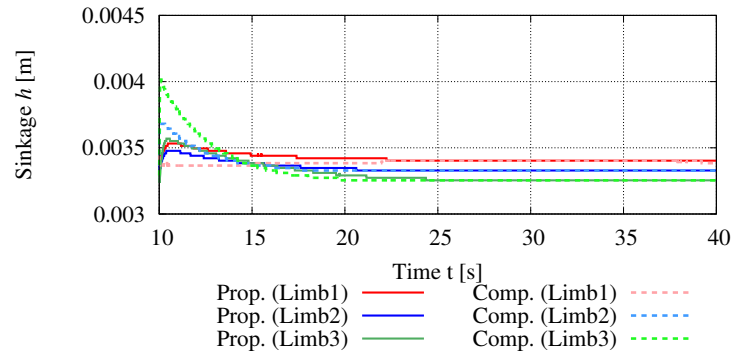

(f) Wheel sinkage $h$.

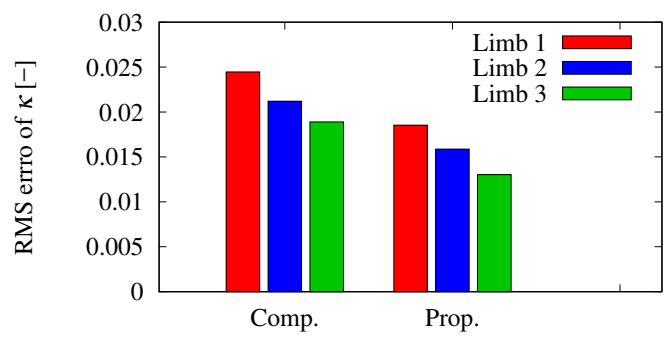

(g) RMS of slip ratio $\kappa$.

Figure 4. Simulation results. 